\title{
BMJ Open Does robotic-assisted unicompartmental knee arthroplasty have lower complication and revision rates than the conventional procedure? A systematic review and meta-analysis
}

\author{
Yifeng Sun, ${ }^{1}$ Wei Liu, ${ }^{1}$ Jian Hou, ${ }^{2}$ Xiuhua Hu, ${ }^{1}$ Wenqiang Zhang (D) ${ }^{1}$
}

To cite: Sun Y, Liu W, Hou J, et al. Does robotic-assisted unicompartmental knee arthroplasty have lower complication and revision rates than the conventional procedure? A systematic review and meta-analysis. BMJ Open 2021;11:e044778. doi:10.1136/ bmjopen-2020-044778

- Prepublication history and additional supplemental material for this paper are available online. To view these files, please visit the journal online (http://dx.doi.org/10.1136/ bmjopen-2020-044778)

Received 12 September 2020 Accepted 12 July 2021

Check for updates

(C) Author(s) (or their employer(s)) 2021. Re-use permitted under CC BY-NC. No commercial re-use. See rights and permissions. Published by BMJ.

${ }^{1}$ Department of Orthopedic Surgery, The First Affiliated Hospital of Shandong First Medical University \& Shandong Provincial Qianfoshan Hospital, Shandong Key Laboratory of Rheumatic Disease and Translational Medicine, Jinan, People's Republic of China ${ }^{2}$ Emergency Department, Jimo Traditional Chinese Hospital, Qingdao, People's Republic of China

Correspondence to Dr Wenqiang Zhang; qfszwq@sina.com

\section{ABSTRACT}

Objective We conducted this systematic review and meta-analysis of studies on patients who underwent unicompartmental knee arthroplasty (UKA) to compare the complication rates, revision rates and non-implant-specific complications between robotic-assisted and conventional UKA.

Design Systematic review and meta-analysis.

Data sources The PubMed, Embase, Web of Science and Cochrane databases were searched up to 30 June 2020. Eligibility criteria Case-control studies comparing robotic-assisted and conventional UKA.

Data extraction and synthesis Data from all eligible articles were independently extracted by two authors. We analysed the differences in outcomes between robotic-assisted and conventional UKA by calculating the corresponding $95 \% \mathrm{Cls}$ and pooled relative risks (RRs). Heterogeneity was assessed using the $\chi^{2}$ and $\mathrm{I}^{2}$ tests. All analyses were performed using the 'metafor' package of $\mathrm{R}$ V.3.6.2 software.

Results A total of 16 studies involving 50024 patients were included in the final meta-analysis. We found that robotic-assisted UKA had fewer complications (RR: $0.52,95 \% \mathrm{Cl}: 0.28$ to $0.96, \mathrm{p}=0.036$ ) and lower revision rates (RR: $0.42,95 \% \mathrm{Cl}: 0.20$ to $0.86, \mathrm{p}=0.017$ ) than conventional UKA. We observed no significant differences in non-implant-specific complications between the two surgical techniques (RR: $0.80,95 \% \mathrm{Cl}: 0.61$ to 1.04 , $\mathrm{p}=0.96$ ). No publication bias was found in this metaanalysis.

Conclusions This study provides evidence that roboticassisted UKA has fewer complications and lower revision rates than conventional UKA; however, owing to important limitations, the results lack reliability, and more studies are required.

PROSPERO registration number CRD42021246927.

\section{INTRODUCTION}

Unicompartmental knee arthroplasty (UKA) is often performed for treating isolated compartmental knee osteoarthritis owing to its minimally invasive nature and less bone resection required during surgery.
Strengths and limitations of this study

$\Rightarrow$ We conducted a meta-analysis to find the best evidence comparing robotic-arm-assisted and manual unicompartmental knee arthroplasty.

$\Rightarrow$ Long-term complications and revision rates depend on the follow-up duration; however, all included studies had a short follow-up period (3-60 months). Hence, the data on revision rates are not reliable.

$\Rightarrow$ Some studies were not randomised controlled trials and had a small sample size, which increase the possibility of publication bias.

$\Rightarrow$ The relatively modest sample size might have caused an unavoidable risk of bias.

$\Rightarrow$ Our results were not adjusted for other factors that could influence outcomes related to knee function, such as patient age and weight, anterior cruciate ligament status, soft tissue balance and composition and thickness of the polyethylene component.

However, higher rates of revision surgery $(10 \%-20 \%)$ have been reported in patients undergoing UKA than in those undergoing total knee arthroplasty. ${ }^{1}$ There could be multiple reasons for the higher failure rate, including poor patient selection and component design, whereas some authors have identified malpositioning as the cause. ${ }^{2}$ The use of robotic systems, which offer promising short-term radiological outcomes of implants and precision in bone cuts, during UKA has considerably increased. Currently, approximately $15 \%-20 \%$ of UKA surgeries are being performed with the assistance of robotic systems, with improved clinical efficacy. ${ }^{3}$ Most experts believe that robotic-assisted UKA provides significantly better component angle alignment accuracy and functional outcomes, as well as higher patient satisfaction, than conventional UKA. However, there are considerable variations in the complication 
and revision rates reported in previous studies, which make it difficult to estimate the safety outcomes of the two surgical techniques. ${ }^{45}$

Previous meta-analyses have compared the effects and safety of robotic-assisted and conventional UKA. In a meta-analysis by $\mathrm{Fu}$ et al, it was reported that roboticassisted UKA showed no decrease in the rate of adverse events compared with conventional UKA. However, few articles (only seven studies) were included in the meta-analysis, and the difference in the revision rates between the two techniques was not reported. ${ }^{6}$ Another meta-analysis by Zhang et al contradicted the conclusion about adverse events by $\mathrm{Fu} e t$ al, reporting instead that robotic-assisted UKA could significantly reduce the rate of complications; however, the results were also subject to limitations in sample size and follow-up duration, which might influence the assessment of the difference in outcomes between robotic-assisted and conventional UKA. ${ }^{4}$ Another recent meta-analysis did not reach a definitive conclusion about complications. ${ }^{5}$ Therefore, we conducted this systematic review and meta-analysis of studies on patients who underwent UKA to compare the complication rates, revision rates and non-implantspecific complications between robotic-assisted and conventional UKA. We hypothesised that there would be no obvious differences in complication and revision rates between the two techniques.

\section{METHODS}

\section{Search strategy}

We searched the PubMed, Web of Science, Embase and Cochrane databases using combinations of the following keywords: 'unicompartmental knee arthroplasty', 'UKA', 'conventional UKA', 'traditional UKA', 'manual UKA', 'robotic-assisted UKA', 'non-robotically assisted UKA', 'complications', 'adverse events' and 'revision' (last updated on 30 June 2020). The references of the identified reports were also retrieved and reviewed to find other related studies. All studies were carefully and repeatedly evaluated. The study period, treatment information, hospital and any additional inclusion criteria were used to identify duplicate or overlapping data.

\section{Inclusion and exclusion criteria}

Studies that met the following criteria were considered eligible for inclusion in this study: (1) original studies about UKA, (2) studies that compared robotic-assisted and conventional UKA, (3) studies that provided controls and effective data (including randomised controlled trials (RCTs), prospective cohort trials, case-control studies and retrospective comparative studies) and (4) studies published in English. The exclusion criteria were as follows: (1) studies published as talks, reviews, digests, letters, commentaries or case reports; (2) model-based or cadaver studies; (3) duplicate or overlapping studies; and (4) not case-control studies.

\section{Data extraction and quality assessment}

The data from all eligible articles were independently extracted by two authors, who discussed any disagreements to reach a consensus. The data retrieved from each study included the first author's name, year of publication, country, methods, number of patients, follow-up duration, complications, revision rate and non-implantspecific complications. Three experienced reviewers used the modified Newcastle-Ottawa quality assessment scale to evaluate the quality of the selected studies. A score of 9 was assigned to studies of superior quality, between 6 and 8 to high-quality studies, between 3 and 5 to moderatequality studies and $<3$ to low-quality studies. ${ }^{7}$

\section{Statistical analysis}

We analysed the differences in outcomes between robotic-assisted and conventional UKA by calculating the corresponding 95\% CIs and pooled relative risks (RRs). Heterogeneity was assessed using the $\chi^{2}$ and $\mathrm{I}^{2}$ tests. Fixed-effect models were employed when there was no significant heterogeneity $\left(\mathrm{I}^{2} \leq 50 \%, \mathrm{p}>0.10\right)$; otherwise, a random-effects model was used to obtain the pooled effects among the included studies. Galbraith plots were used to detect potential sources of heterogeneity. ${ }^{8}$ Normal quantile-quantile ( $Q-Q)$ plots were used to check for deviation of data from the CI. Outlier and influence analyses were performed by inspecting the plots for externally standardised residues, DFFITS values, Cook's distances, covariance ratios, estimates of $\tau^{2}$, test statistics for residual heterogeneity when each study was excluded in turn, hat values, and weights for each study included in the analysis. ${ }^{9}$ Publication bias was assessed by inspecting a contour-enhanced funnel plot, with contours at $90 \%$, 95\% and $99 \%$ CIs. All analyses were performed using the 'metafor' package of R V.3.6.2 software. ${ }^{10}$ A two-tailed p value of $<0.05$ was considered statistically significant.

\section{Patient and public involvement}

There was no patient and public involvement in this systematic review.

\section{RESULTS}

\section{Study characteristics}

We initially identified 374 studies through the search of the PubMed, Embase, Web of Science and Cochrane databases. Of these, 322 studies did not meet the inclusion criteria and were excluded after reviewing the titles and abstracts. Of the 52 remaining studies that were subjected to a full-text review, 28 were excluded because they were not comparative studies. In addition, eight full-text articles were excluded for the following reasons: (1) data were incomparable or incomplete and (2) data about complications were not available. Finally, 16 studies involving 50024 patients were included in the final metaanalysis. The flow diagram of study selection is presented in figure 1. Table 1 summarises the main characteristics of the 16 included studies. The quality assessment of the 


\section{4studies were identified base on the search criteria}

Excluded 322 studies by title and abstract, with reasons:

(1) Type of literature specified as a talk, review, digest, letter, commentary, digest,or case report;

(2) Model-based or cadaver studies;

(3) Duplicate or overlapping data

52 remaining studies for full text review

Excluded 28 studies due to not comparison trials

24 full-text articles assesed for eligibility

Excluded 8 full-text articles, with reasons:

(1) Data were incomparable or incomplete

(2) Have no complication results

16 studies were included in this meta-analysis

Figure 1 Flow diagram depicting the study selection procedure.

included studies is presented in detail in the supplementary material, and all the studies were evaluated as being of moderate-to-high quality (online supplemental table S1).

\section{Complications}

Complications that lead to failure of UKA include bearing dislocation, aseptic loosening, polyethylene wear, periprosthetic fracture, progression of arthritis to the contralateral compartment, infection, bone-implant impingement, retained cement debris in the joint, knee ankylosis, wound complications, deep haematoma, infection, thrombosis, persistent pain, pin-site infection and fracture and other adverse events. All 16 studies reported data about complications, which mainly involved prosthetic loosening, subsidence, polyethylene bearing dislocation, periprosthetic fracture, knee ankylosis, wound complications, deep haematoma, infection, thrombosis and persistent pain. The $\chi^{2}$ and $\mathrm{I}^{2}$ test results showed statistical heterogeneity between the included studies $\left(\mathrm{p}<0.01, \mathrm{I}^{2}=87.1 \%\right)$, and Galbraith plots showed that no single study caused heterogeneity (figure $2 \mathrm{~A}$ ). The plotted points were close to a sloped straight line on the Q-Q plot (figure 2B), which showed that there was no significant deviation from the CI in the included studies. Therefore, a random-effects model was used for the analysis. We found that robotic-assisted UKA had a lower rate of complications than conventional UKA (RR: 0.52, 95\% CI: 0.28 to $0.96, \mathrm{p}=0.0366$; figure $2 \mathrm{C}$ ).

\section{Revision rate}

Ten studies reported data about complications that required surgery in the two groups. The $\chi^{2}$ and $\mathrm{I}^{2}$ test results showed statistical heterogeneity among the included studies $\left(\mathrm{p}<0.01, \mathrm{I}^{2}=90.3 \%\right)$ and Galbraith plots were used to determine the most heterogeneous studies; however, no studies were excluded (figure 3A). As seen from the $Q-Q$ plot, there was no significant deviation from the $\mathrm{CI}$ in the studies (figure 3B). Data pooled using a random-effects model indicated that robotic-assisted UKA had a lower rate of revision surgery (RR: 0.42, 95\% CI: 0.20 to $0.86, \mathrm{p}=0.017$; figure $3 \mathrm{C}$ ).

\section{Non-implant-specific complications}

Non-implant-specific complications were reported in 10 studies, which mainly involved infection, knee ankylosis, wound complications, deep haematoma, infection, thrombosis, persistent pain and pin-site infection and fracture in our meta-analysis. The $\chi^{2}$ and $\mathrm{I}^{2}$ test results indicated no statistical heterogeneity among the included studies $\left(\mathrm{p}=0.49, \mathrm{I}^{2}=0.00 \%\right)$ and Galbraith plots (figure $\left.4 \mathrm{~A}\right)$ and Q-Q plots (figure 4B) also showed no statistical heterogeneity. We observed no significant differences in nonimplant-specific complications between the two groups in comparisons using a fixed-effects model (RR: 0.80, 95\% CI: 0.61 to $1.04, \mathrm{p}=0.96$; figure $4 \mathrm{C}$ ).

\section{Publication bias}

We assessed publication bias using Begg's test. ${ }^{11}$ The contour-enhanced funnel plot for the meta-analysis of complications of robotic-assisted versus conventional UKA was largely symmetric $\left(\mathrm{P}_{\mathrm{Begg}}=0.96\right.$; figure $\left.5 \mathrm{~A}\right)$. Similar results were observed for the revision rate $\left(\mathrm{P}_{\mathrm{Begg}}=0.78\right.$; figure 5B) and non-implant-specific complications $\left(\mathrm{P}_{\text {Begg }}=1.16\right.$; figure 5C).

\section{Outlier and influence analyses}

The presence of outliers and influential studies can affect the validity and robustness of conclusions from a meta-analysis. Figure 5 shows the standardised residuals (rstudent), DFFITS (dffits), Cook's distances (cook.d), covariance ratios (cov.r), estimates of $\tau^{2}$ (tau2.del) and test statistics (QE.del) for the random-effects model that was used for the analysis of complications (figure 6). The study by Vakharia et al was identified as a potential outlier that led to heterogeneity and seemed to be an influential study. As the study had a large sample size (35061 patients, robotic-assisted=13617; conventional=21444), making it useful for analysing national trends, and the hat values and weights showed that this study comprised the largest proportion of patients in the meta-analysis, it was not excluded from the meta-analysis. A similar result was observed in the analysis of the revision rate. No outlier was included in the analysis of non-implant-specific complications.

\section{DISCUSSION}

For more than 50 years, UKA has been performed to treat isolated compartmental knee arthritis. Despite many years of experience in performing UKA, some studies have reported that UKA has higher rates of failure than total knee arthroplasty. ${ }^{12}$ Newly designed roboticassisted systems are believed to increase the precision 

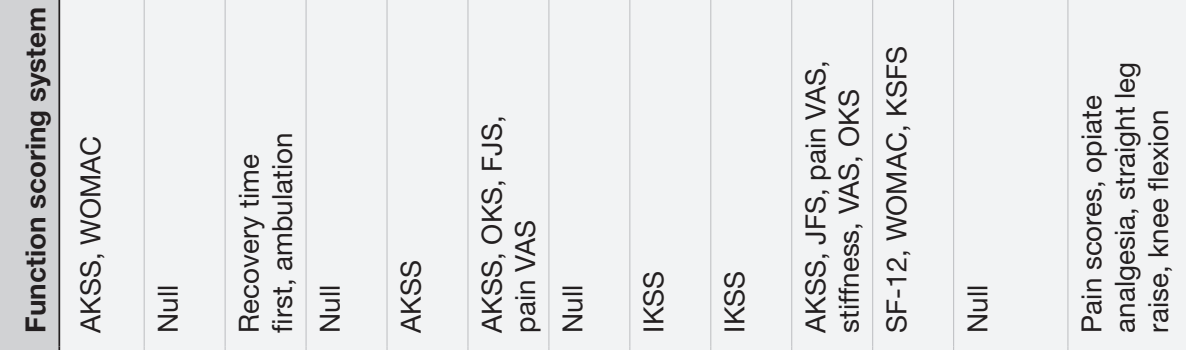

管彭言量

列耪言

\section{辤喜}

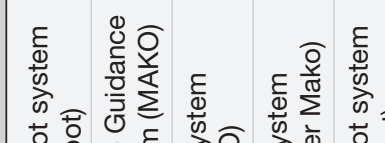

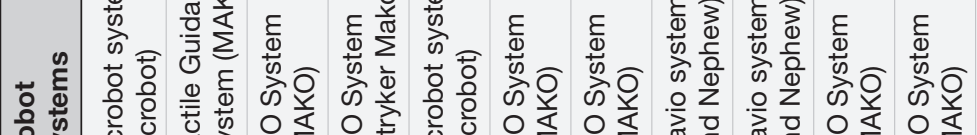

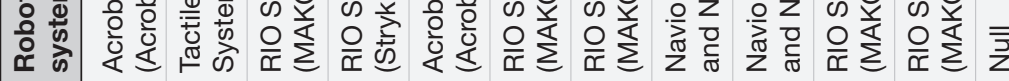

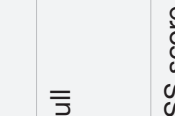

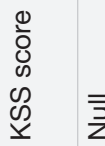

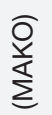

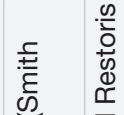

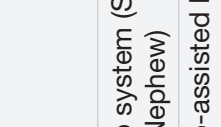

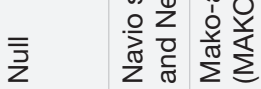

$\frac{2}{\frac{0}{9}}$

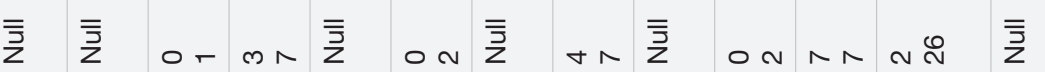

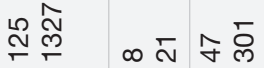

$-$

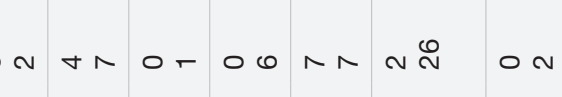

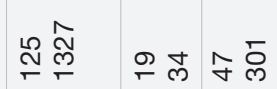

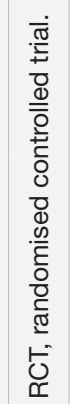

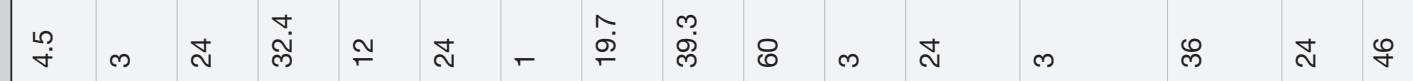

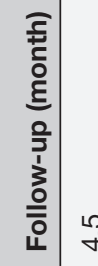

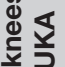



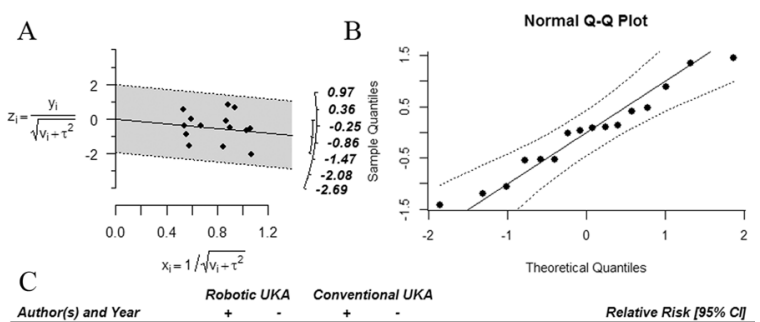

Conventional UKA Theoretical Quartiles

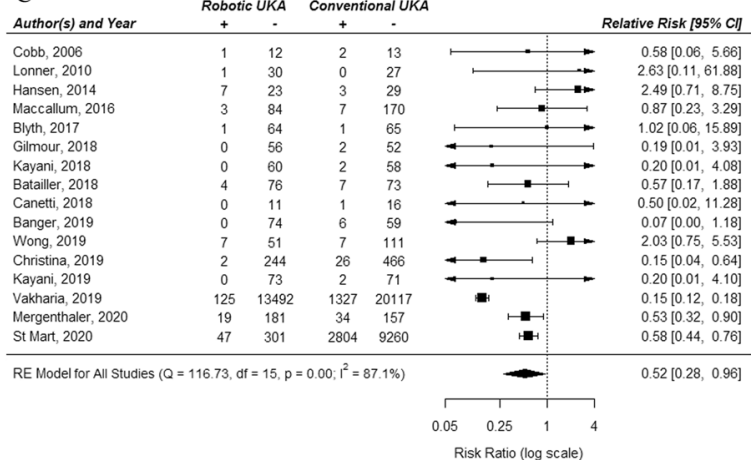

Figure 2 (A) Galbraith plot, (B) quantile-quantile (Q-Q) plot and (C) forest plot for the comparison of complications between robotic-assisted and conventional unicompartmental knee arthroplasty (UKA).

and accuracy of UKA, possibly leading to fewer complications and lower revision rates. ${ }^{13}$ Many studies have evaluated the complications of robotic-assisted UKA; however, there are few studies on the complications of robotic-assisted UKA compared with those with conventional UKA. Researchers have reported conflicting results about the complication rates between robotic-assisted and conventional UKA. Hansen et al and Blyth et al did not find a significant difference in the rate of complications between the two techniques. ${ }^{14} 15$ Wong et al found that the robotic-arm-assisted arthroplasty cohort had a higher early revision rate than the conventional group, whereas other studies reported that robotic-assisted UKA had fewer complications and lower revision rates than conventional UKA. ${ }^{16-18}$ It is important to assess the complications of this new technology before it can be widely used. ${ }^{19}$ Therefore, we conducted this systematic review and meta-analysis to compare the complication rates, revision rates and non-implant-specific complications between robotic-assisted and conventional UKA. The main finding of our meta-analysis was that roboticassisted UKA has fewer complications and lower revision rates than conventional UKA; however, there were no significant differences in non-implant-specific complications. Thus, our study confirms that robotic-assisted UKA has fewer complications and lower revision rates than the conventional procedure.

Many studies have explored the relationship between the component position and its impact on implant survival and patient satisfaction. ${ }^{2021}$ Some authors believe that a reduction in alignment errors of these components will ultimately affect implant function or survival. ${ }^{22}$ Some studies confirmed that the proportion of patients with tibial and femoral component implantation within $2^{\circ}$ of the target position was significantly greater in the group
A

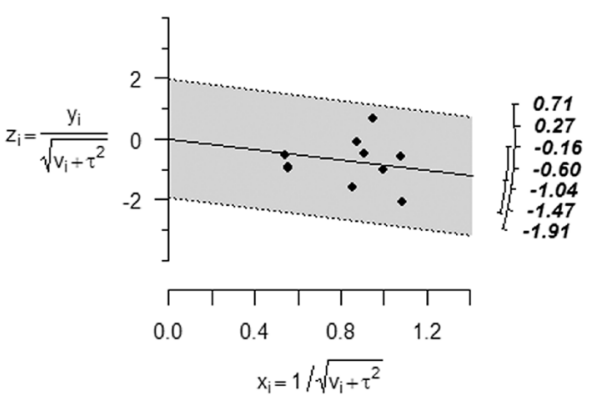

B

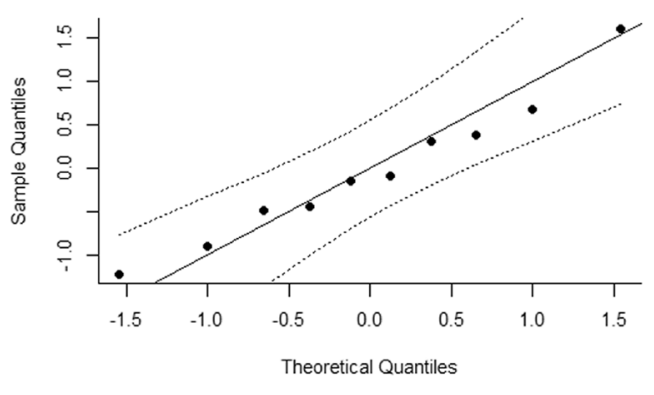

\begin{tabular}{|c|c|c|c|c|c|c|c|}
\hline \multirow{2}{*}{$\begin{array}{l}\text { C } \\
\text { Author(s) and Year }\end{array}$} & \multicolumn{2}{|c|}{ Robotic UKA } & \multicolumn{2}{|c|}{ Conventional UKA } & & & \multirow[b]{2}{*}{ Relative Risk [ $[95 \% \mathrm{Cl}]$} \\
\hline & + & - & + & - & & & \\
\hline Hansen, 2014 & 0 & 30 & 1 & 31 & 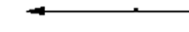 & - & $0.35[0.02,8.39]$ \\
\hline Maccallum, 2016 & 3 & 84 & 7 & 170 & & $\longrightarrow$ & $0.87[0.23,3.29]$ \\
\hline Gilmour, 2018 & 0 & 56 & 2 & 52 & - & & $0.19[0.01,3.93]$ \\
\hline Batailler, 2018 & 4 & 76 & 7 & 73 & & $\longrightarrow$ & $0.57[0.17,1.88]$ \\
\hline Banger, 2019 & 0 & 74 & 2 & 63 & $\rightarrow$ & $一$ & $0.18[0.01,3.60]$ \\
\hline Wong, 2019 & 7 & 51 & 7 & 111 & & $\rightarrow-$ & $2.03[0.75,5.53]$ \\
\hline Christina, 2019 & 2 & 244 & 26 & 466 & & & $0.15[0.04,0.64]$ \\
\hline Vakharia, 2019 & 125 & 13492 & 1327 & 20117 & 淂 & & $0.15[0.12,0.18]$ \\
\hline Mergenthaler, 2020 & 8 & 192 & 21 & 170 & $\longmapsto$ & & $0.36[0.17,0.80]$ \\
\hline St Mart, 2020 & 47 & 301 & 2804 & 9260 & 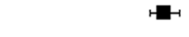 & & $0.58[0.44,0.76]$ \\
\hline \multirow{3}{*}{\multicolumn{5}{|c|}{ RE Model for All Studies $\left(Q=93.24, \mathrm{df}=9, p=0.00 ; I^{2}=90.3 \%\right)$}} & & & $0.42[0.20,0.86]$ \\
\hline & & & & & 1 & ᄀ & \\
\hline & & & & & 0.05 & 1 & \\
\hline
\end{tabular}

Figure 3 (A) Galbraith plot, (B) quantile-quantile (Q-Q) plot and (C) forest plot for the comparison of revision rate between robotic-assisted and conventional unicompartmental knee arthroplasty (UKA). 
A

B

Normal Q-Q Plot

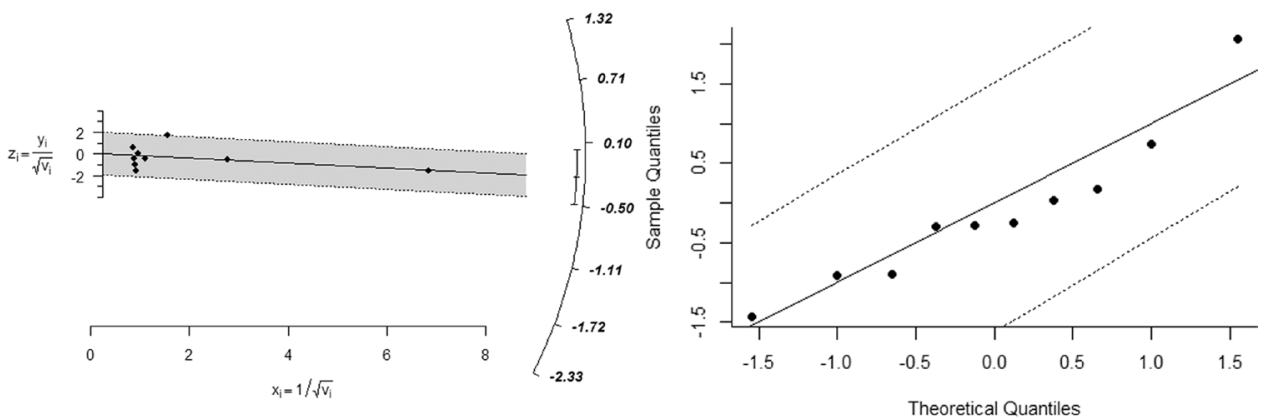

$\mathrm{C}$

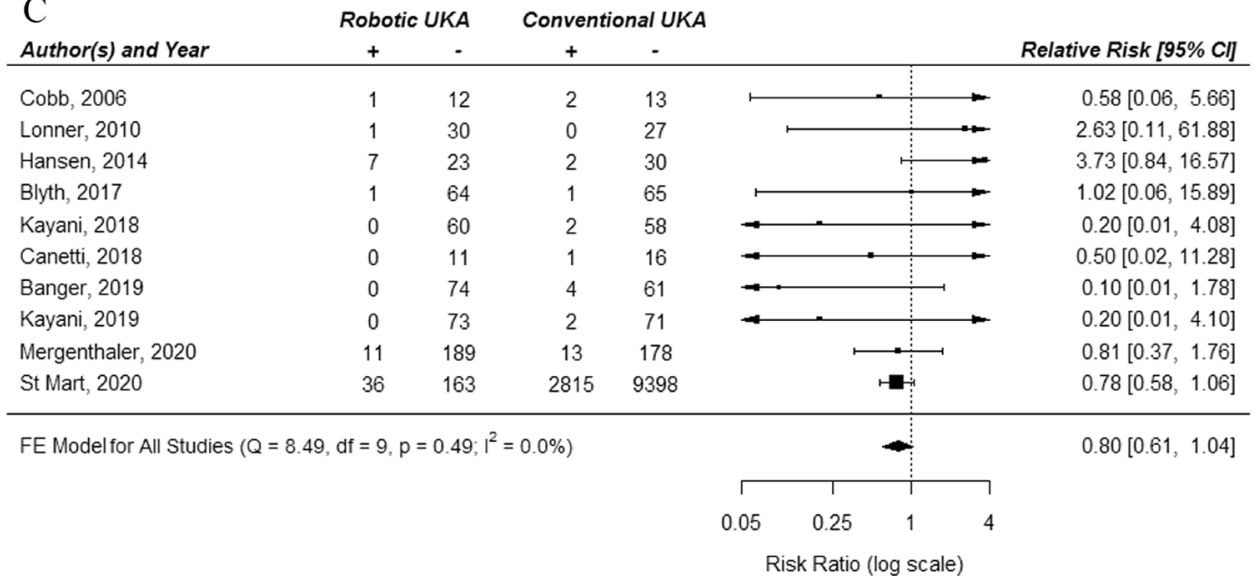

Figure 4 (A) Galbraith plot, (B) quantile-quantile (Q-Q) plot and (C) forest plot for the comparison of non-implant-specific complications between robotic-assisted and conventional unicompartmental knee arthroplasty (UKA).
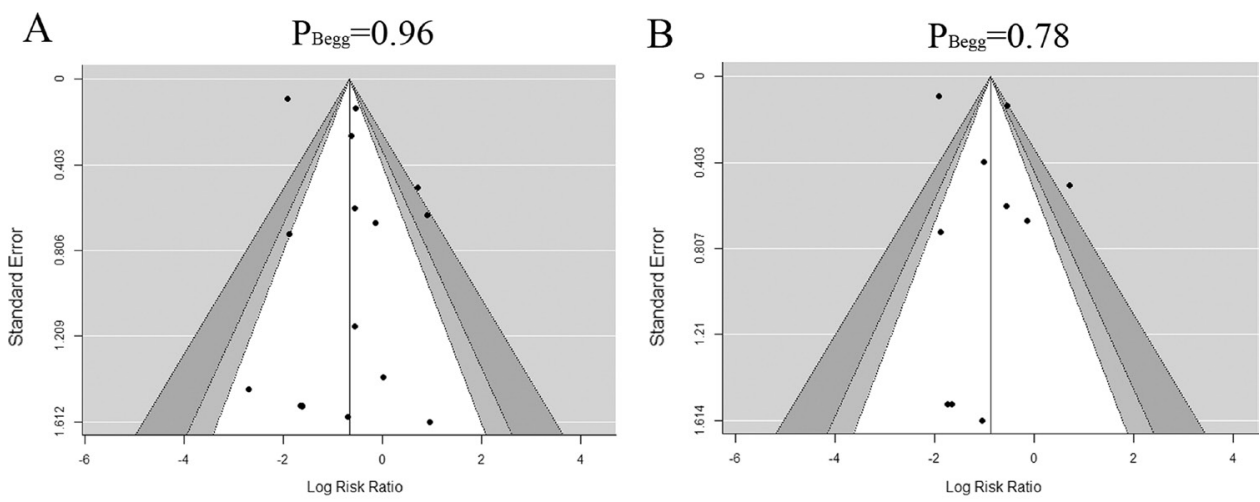

$\mathrm{C}$

$\mathrm{P}_{\text {Begg }}=1.16$

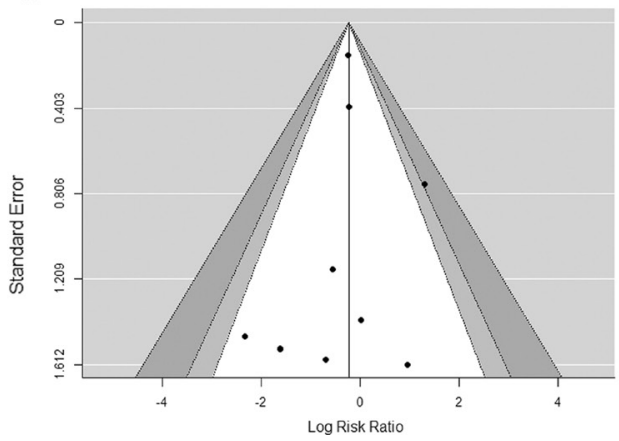

Figure 5 Contour-enhanced funnel plots of the included studies showing no evidence of publication bias in complications (A), revision rate (B) and non-implant-specific complications (C). 

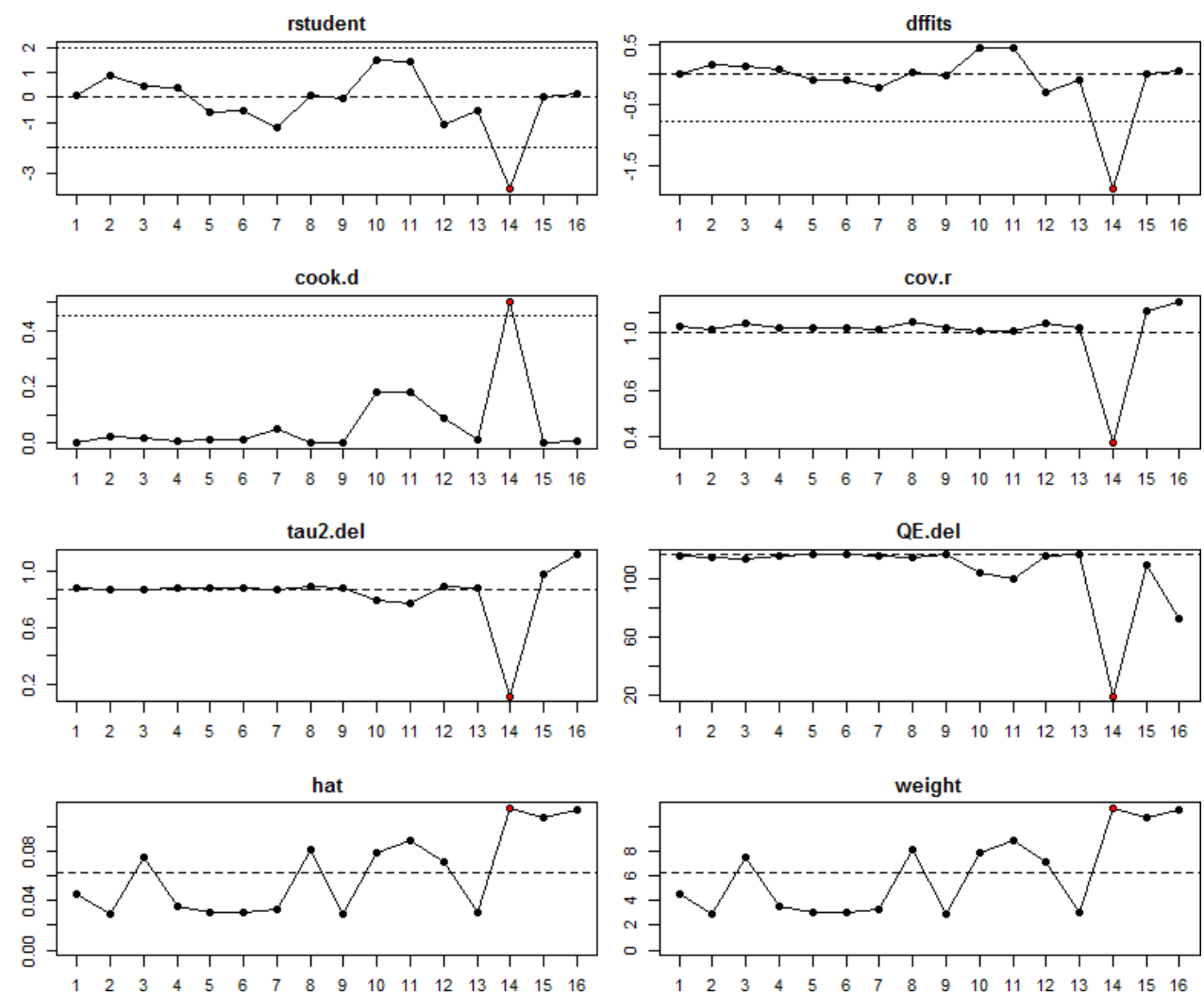

Figure 6 Outlier and influence analyses. The standardised residuals (rstudent), DFFITS (dffits), Cook's distances (cook.d), covariance ratios (cov.r), estimates of $\tau^{2}$ (tau2.del) and test statistics (QE.del) for the random-effects model that was used for the analysis of the complications are shown.

that underwent robotic-assisted UKA, resulting in better long-term clinical scores and a lower implant failure rate. ${ }^{2024}$ Therefore, it could be inferred that the use of a robotic-assisted system in UKA can reduce implantation errors, leading to fewer complications and lower rates of revision surgery than conventional UKA. Although nonimplant-specific complications are likely to be related to the procedure, fewer complications were considered to be directly related to the comparative study itself. ${ }^{25}$ Mergenthaler et al reported no complications related to the use of the robotic system. ${ }^{26}$ Pearle $e t$ al suggested that no further rigid fixation device is necessary, which reduces potential complications such as infection, iatrogenic fractures or soft tissue injury caused by the weight and movement of the robot. ${ }^{27}$ However, there were no significant differences in non-implant-specific complications between the two techniques in our meta-analysis. Therefore, there is no evidence that the use of robotic systems can add to the non-implant-specific complications of UKA.

Although robotic-assisted UKA is widely performed and is the current trend in orthopaedic surgery, it has some shortcomings. Robotic-assisted UKA was found to significantly prolong the duration of surgery compared with conventional UKA (online supplemental figure S1). Some studies have also documented that roboticarm-assisted UKA has a higher cost. ${ }^{28}{ }^{29}$ In addition, the device-related complications, such as pin-site fracture and infection, are non-negligible. We checked all articles included in the meta-analysis for a statement on funding or conflicts of interest related to the work. When such a statement was provided, we categorised the study as an industry-funded study or involving authors with financial conflicts of interest. We found that the included articles were more likely to be industry funded or written by authors with financial conflicts of interest (online supplemental figure S2). Therefore, this information should not be overlooked, and more large-scale, non-commercially supported studies evaluating the efficacy of the two treatments in this patient population are needed in the future.

This meta-analysis has several limitations. First, longterm revision rates depend on the duration of follow-up; however, all included studies had a short follow-up period (3-60 months). Hence, the data on revision rates are not reliable. Future studies with a longer follow-up duration, preferably 10 years, are necessary to assess complications and revision rates. Second, some studies were not RCTs and had a small sample size, which increase the possibility of publication bias. Therefore, our results should be further confirmed by large-scale RCTs. Third, the types of robotic-assisted UKA performed in each study were different, as shown in table 1 . The different types of robotic systems used were the Acrobot, RIO or Makoassisted Restoris and Navio systems. Rapid advances in robotic-assisted technology have led to improvements in UKA over the past 10 years. Implant position, soft tissue balance and radiographic component alignment 
seem to have gradually improved with the development of robotic-assisted systems. Considering the evolution of this technology and its possible impact on outcomes, well-designed studies are necessary to advance the understanding of the impact of different robotic systems. Fourth, all included studies were limited to the English literature; therefore, some related studies published in other languages that might have met the inclusion criteria could have been missed. Fifth, most of the studies in our meta-analysis did not report pin-site and devicespecific complications. Revision surgeries secondary to pin-site fracture were reported in some studies; however, the sample size was small. Therefore, we did not conduct a systematic analysis on these specific complications and revisions. Although we attempted to identify and retrieve all additional unpublished information, some missing data were inevitable. In addition, our results were not adjusted for other factors that could influence complications, such as patient age and weight, anterior cruciate ligament status, soft tissue balance and composition and thickness of the polyethylene component. Sixth, some of the included studies did not mention the reasons for loss to follow-up or lack details about revision surgery. However, these might have no effect on the analysis. Finally, when events such as complications and revisions occur over a non-fixed period, it is common to use HRs as the statistic of interest. As the 'metafor' package has no function for using HRs as the statistic of interest, we used RRs as the statistic of interest across all studies.

\section{CONCLUSIONS}

To summarise, this meta-analysis study indicates that robotic-assisted UKA is associated with fewer complications and lower rates of revision surgery than conventional UKA. No evidence suggests that the use of robotic systems might increase the rate of non-implant-specific complications of UKA.

Therefore, the study provides evidence that roboticassisted UKA has fewer complications and lower revision rates than conventional UKA; however, owing to important limitations, the results lack reliability, and more studies are required.

Contributors YS conceived the study and wrote the manuscript. WZ analysed the data. WL generated data. $\mathrm{XH}$ and $\mathrm{JH}$ reviewed the manuscript.

Funding This work was supported by the Natural Science Foundation of China (81702667), Wu Jieping Medical Foundation (320.6750.18466) and Shandong Provincial Key Research and Development Project (2019GSF108112).

Competing interests None declared.

Patient consent for publication Not required.

Ethics approval This study is a systematic review and meta-analysis, there was no ethics committee(s) or institutional board(s) involvement in this study.

Provenance and peer review Not commissioned; externally peer reviewed.

Data availability statement No data are available. All data relevant to the study are included in the article or uploaded as supplemental information.

Supplemental material This content has been supplied by the author(s). It has not been vetted by BMJ Publishing Group Limited (BMJ) and may not have been peer-reviewed. Any opinions or recommendations discussed are solely those of the author(s) and are not endorsed by BMJ. BMJ disclaims all liability and responsibility arising from any reliance placed on the content. Where the content includes any translated material, BMJ does not warrant the accuracy and reliability of the translations (including but not limited to local regulations, clinical guidelines, terminology, drug names and drug dosages), and is not responsible for any error and/or omissions arising from translation and adaptation or otherwise.

Open access This is an open access article distributed in accordance with the Creative Commons Attribution Non Commercial (CC BY-NC 4.0) license, which permits others to distribute, remix, adapt, build upon this work non-commercially, and license their derivative works on different terms, provided the original work is properly cited, appropriate credit is given, any changes made indicated, and the use is non-commercial. See: http://creativecommons.org/licenses/by-nc/4.0/.

\section{ORCID iD}

Wenqiang Zhang http://orcid.org/0000-0001-5584-2588

\section{REFERENCES}

1 Labek G, Sekyra K, Pawelka W. Outcome and reproducibility of data concerning the Oxford medial Unicompartmental knee arthroplasty. Orthopaedic Proceedings 2011;93:131.

2 Lang JE, Mannava S, Floyd AJ, et al. Robotic systems in orthopaedic surgery. J Bone Joint Surg Br 2011;93:1296-9.

3 Lonner JH, Moretti VM. The evolution of Image-Free robotic assistance in Unicompartmental knee arthroplasty. Am J Orthop 2016;45:249-54.

4 Zhang F, Li H, Ba Z, et al. Robotic arm-assisted vs conventional unicompartmental knee arthroplasty: a meta-analysis of the effects on clinical outcomes. Medicine 2019;98:e16968.

5 Chin BZ, Tan SSH, Chua KCX, et al. Robot-assisted versus conventional total and unicompartmental knee arthroplasty: a meta-analysis of radiological and functional outcomes. J Knee Surg 2020. doi:10.1055/s-0040-1701440. [Epub ahead of print: 17 Mar 2020].

6 Fu J, Wang Y, Li X, et al. Robot-assisted vs. conventional unicompartmental knee arthroplasty : systematic review and metaanalysis. Orthopade 2018;47:1009-17.

7 Wells G. The newcastle-ottawa scale (NOS) for assessing the quality of non-randomised studies in meta-analyses. In: Symposium on systematic reviews: beyond the basics, 2014

8 Galbraith RF. Some applications of radial plots. J Am Stat Assoc 1994;89:1232-42.

9 Viechtbauer W, Cheung MW-L. Outlier and influence diagnostics for meta-analysis. Res Synth Methods 2010;1:112-25.

10 Viechtbauer W. Conducting Meta-Analyses in $R$ with the metafor Package. J Stat Softw 2010;36:1-48.

11 Macaskill P, Walter SD, Irwig L. A comparison of methods to detect publication bias in meta-analysis. Stat Med 2001;20:641-54.

12 Sun X, Su Z. A meta-analysis of unicompartmental knee arthroplasty revised to total knee arthroplasty versus primary total knee arthroplasty. J Orthop Surg Res 2018;13:158.

13 Roche M. Robotic-assisted unicompartmental knee arthroplasty: the MAKO experience. Clin Sports Med 2014;33:123-32.

14 Hansen DC, Kusuma SK, Palmer RM, et al. Robotic guidance does not improve component position or short-term outcome in medial Unicompartmental knee arthroplasty. J Arthroplasty 2014;29:1784-9.

15 Blyth MJG, Anthony I, Rowe P, et al. Robotic arm-assisted versus conventional unicompartmental knee arthroplasty: exploratory secondary analysis of a randomised controlled trial. Bone Joint Res 2017;6:631-9.

16 Wong J, Murtaugh T, Lakra A, et al. Robotic-assisted unicompartmental knee replacement offers no early advantage over conventional unicompartmental knee replacement. Knee Surg Sports Traumatol Arthrosc 2019;27:2303-8.

17 Vakharia RM, Sodhi N, Cohen-Levy WB, et al. Comparison of patient demographics and utilization trends of robotic-assisted and nonrobotic-assisted unicompartmental knee arthroplasty. J Knee Surg 2021;34:621-7.

18 St Mart J-P, de Steiger RN, Cuthbert A, et al. The three-year survivorship of robotically assisted versus non-robotically assisted unicompartmental knee arthroplasty. Bone Joint J 2020;102B:319-28.

19 Cool CL, Needham KA, Khlopas A, et al. Revision analysis of robotic arm-assisted and manual unicompartmental knee arthroplasty. $J$ Arthroplasty 2019;34:926-31. 
20 Gromov K, Korchi M, Thomsen MG, et al. What is the optimal alignment of the tibial and femoral components in knee arthroplasty? Acta Orthop 2014;85:480-7

21 Vandekerckhove P-J, Lanting B, Bellemans J, et al. The current role of coronal plane alignment in total knee arthroplasty in a preoperative varus aligned population: an evidence based review. Acta Orthop Belg 2016;82:129-42.

$22 \mathrm{Kim} \mathrm{KT}$, Lee S, Kim TW, et al. The influence of postoperative tibiofemoral alignment on the clinical results of unicompartmental knee arthroplasty. Knee Surg Relat Res 2012;24:85-90.

23 MacCallum KP, Danoff JR, Geller JA. Tibial baseplate positioning in robotic-assisted and conventional unicompartmental knee arthroplasty. Eur J Orthop Surg Traumatol 2016;26:93-8.

24 Chatellard R, Sauleau V, Colmar M, et al. Medial unicompartmental knee arthroplasty: does tibial component position influence clinica outcomes and arthroplasty survival? Orthop Traumatol Surg Res 2013;99:S219-25.

25 Cobb J, Henckel J, Gomes P, et al. Hands-on robotic unicompartmental knee replacement: a prospective, randomised controlled study of the acrobot system. J Bone Joint Surg $\mathrm{Br}$ 2006;88:188-97.

26 Mergenthaler G, Batailler C, Lording T, et al. Is robotic-assisted unicompartmental knee arthroplasty a safe procedure? A case control study. Knee Surg Sports Traumatol Arthrosc 2021;29:931-8.

27 Pearle AD, O'Loughlin PF, Kendoff DO. Robot-assisted unicompartmental knee arthroplasty. J Arthroplasty 2010;25:230-7.
28 Moschetti WE, Konopka JF, Rubash HE, et al. Can robot-assisted unicompartmental knee arthroplasty be cost-effective? a markov decision analysis. J Arthroplasty 2016;31:759-65.

29 Sinha RK. Outcomes of robotic arm-assisted unicompartmental knee arthroplasty. Am J Orthop 2009;38:20-2

30 Gilmour A, MacLean AD, Rowe PJ. Robotic-arm-assisted vs conventional unicompartmental knee arthroplasty. The 2-year clinical outcomes of a randomized controlled trial. J Arthroplasty 2018;33:S109-15.

31 Kayani B, Konan S, Pietrzak JRT, Huq SS, et al. The learning curve associated with robotic-arm assisted unicompartmental knee arthroplasty: a prospective cohort study. Bone Joint J 2018;100B:1033-42.

32 Batailler C, White N, Ranaldi FM, et al. Improved implant position and lower revision rate with robotic-assisted unicompartmental knee arthroplasty. Knee Surg Sports Traumatol Arthrosc 2019;27:1232-40.

33 Canetti R, Batailler C, Bankhead C, et al. Faster return to sport after robotic-assisted lateral unicompartmental knee arthroplasty: a comparative study. Arch Orthop Trauma Surg 2018;138:1765-71.

34 Banger M, Blyth M, Jones B. 5 year results of a randomised trial of robotic arm assisted vs manual unicompartmental knee arthoplasty, 2019.

35 Kayani B, Konan S, Tahmassebi J, et al. An assessment of early functional rehabilitation and hospital discharge in conventional versus robotic-arm assisted unicompartmental knee arthroplasty: a prospective cohort study. Bone Joint J 2019;101-B:24-33. 